\title{
UNITAL $l$-PRIME LATTICE-ORDERED RINGS WITH POLYNOMIAL CONSTRAINTS ARE DOMAINS
}

BY

STUART A. STEINBERG ${ }^{1}$

To Nathan Jacobson

\begin{abstract}
It is shown that a unital lattice-ordered ring in which the square of every element is positive must be a domain provided the product of two nonzero $l$-ideals is nonzero. More generally, the same conclusion follows if the condition $a^{2} \geqslant 0$ is replaced by $p(a) \geqslant 0$ for suitable polynomials $p(x)$; and if it is replaced by $f(a, b) \geqslant 0$ for suitable polynomials $f(x, y)$ one gets an $l$-domain. It is also shown that if $a \wedge b=0$ in a unital lattice-ordered algebra which satisfies these constraints, then the $l$-ideals generated by $a b$ and $b a$ are identical.
\end{abstract}

1. Introduction. In $[5$, p. 79] Diem has asked if an l-prime l-ring in which the square of every element is positive is an $l$-domain. In this paper we show that any such $l$-ring $R$ is a domain provided the $f$-subring $T$ of $f$-elements has zero annihilator in $R$ or the $T$ - $T$ convex $l$-bimodule of $R$ generated by $T a+a T$ contains $a$ for each nilpotent element $a$ of index 2. Also, some polynomial constraints which generalize the condition that squares are positive are considered, and it is shown that an $l$-prime $l$-ring with such constraints is an $l$-domain, sometimes even a domain. Our original arguments were based on Lemmas 13 and (an earlier version of) 14 . However, while this paper was being revised we realized that the simpler Lemma 2 was sufficient to get $l$-domains from $l$-prime $l$-rings.

A lattice-ordered ring ( $($-ring) is a ring $R$ whose additive group is an $l$-group (that is, $R$ is a lattice and each translation $x \rightarrow a+x$ is order preserving, and hence is an order automorphism) and in which the set of positive elements $R^{+}=\{a \in R$ : $a \geqslant 0\}$ is closed under multiplication. Some good references for background material on $l$-rings are $[4 ; 2 ; 3$, Chapters 13 and $17 ; 6$; 9 , Chapter I, pp. 164-176 and 14 , §2, pp. 192-202]. In particular, in Theorem 1 of [14] and Proposition 1.3 of [9] there is a list of many of the basic equations, inequalities and properties that result from the interaction of the lattice and ring structures in an $l$-ring.

Received by the editors November $15,1981$.

1980 Mathematics Subject Classification. Primary 16A86, 06F25; Secondary 06A12.

Key words and phrases. Lattice-ordering ring, l-prime l-ring, nilpotent element, domain, squares positive, polynomial constraints.

'An earlier version of this paper was presented at the Jacobson Conference at Yale, June, 1981, under the title "Polynomial constraints in lattice-ordered rings". It was also presented to the special session on Ordered Algebraic Structures of the American Mathematical Society on January 15, 1982 at Cincinnati, Ohio. 
The right (left) module $M$ over the $l$-ring $R$ is called an $l$-module if $M$ is an $l$-group and $M^{+} R^{+} \subseteq M^{+}\left(R^{+} M^{+} \subseteq M^{+}\right)$. A convex l-subgroup (submodule) of $M$ is a subgroup (submodule) $X$ that is a sublattice which is also convex: $x \leqslant m \leqslant y$ and $x$, $y \in X$ imply $m \in X$; that is, $X$ is the kernel of an $l$-group ( $l$-module) homomorphism. The element $r \in R^{+}$is an f-element on $M_{R}$ if for all $a, b \in M$

(1) $a \wedge b=0$ implies $a r \wedge b=0$.

If $R^{+}$consists of $f$-elements on $M$, then $M$ is called an $f$-module over $R$. An $l$-module over $R$ is an $f$-module precisely when it is embeddable in a product of totally ordered $R$-modules [13, Theorem 1.1 or 1, p. 54]. Note that when $M_{R}$ is an $f$-module, the map $x \rightarrow x r$ is a lattice homomorphism of $M$ for each $r \in R^{+}$(see, for example [4, Lemma 1, p. 52 or 2 , Theorem 1.4 .4 , p. 25]). If $R$ and $S$ are $l$-rings, then $M$ is an $R-S$ l-bimodule ( $f$-bimodule) if $M$ is a left $l$-module ( $f$-module) over $R$, a right $l$-module ( $f$-module) over $S$ and $r(x s)=(r x) s$ for all $r \in R, x \in M$, and $s \in S$. The $R-S$ $l$-bimodule is an $f$-bimodule if and only if it is embeddable in a product of totally ordered $R-S$ l-bimodules. In particular, $R$ is an $f$-ring (that is, $R$ is an $R-R$ $f$-bimodule) precisely when it is embeddable in a product of totally ordered rings [4, Theorem 12, p. 57]. By an f-element of the l-ring $R$ we mean an element $a \in R^{+}$ which is an $f$-element on both the $l$-modules $R_{R}$ and ${ }_{R} R$. An l-algebra over the commutative unital totally ordered domain $F$ is a ring $R$ which is a torsion-free algebra over $F$ and which is also an $f$-module over $F$. Of course, any $l$-ring $R$ is an $l$-algebra over the integers $\mathbf{Z}$; and if $R$ is also an $l$-module and algebra over the totally ordered field $F$, then it is an $l$-algebra over $F$.

An (right, left) ideal of the $l$-ring $R$ is an (right, left) l-ideal of $R$ if it is also a convex $l$-subgroup of the additive $l$-group of $R . R$ is called $l$-prime if the product of two nonzero $l$-ideals is nonzero, and $R$ is an $l$-domain if the product of two nonzero positive elements is nonzero. $R$ is called (l-reduced) reduced if it has no nonzero (positive) nilpotent elements, and $l$-semiprime if it has no nonzero nilpotent $l$-ideals. Recall that $R$ is $l$-semiprime ( $l$-prime) if and only if for all $a \in R^{+}\left(a, b \in R^{+}\right)$, $a R a=0(a R b=0)$ implies $a=0(a=0$ or $b=0)[5,2.5$, p. 73 or 11]. An $l$-ideal $P$ is an l-prime l-ideal of $R$ if $R / P$ is an l-prime $l$-ring. By the lower l-radical of the $l$-ring $R$ we mean $\beta(R)=$ the intersection of all the $l$-prime $l$-ideals of $R$. The lower $l$-radical is a nil $l$-ideal, and $R$ is $l$-semiprime if and only if $\beta(R)=0[5,2.13$ or 11]. We also note that, just as for rings, an $l$-reduced $l$-prime $l$-ring is an $l$-domain. Birkhoff and Pierce [4, p. 63] have shown:

(2) If $R$ is an $f$-ring, then $N_{n}=\left\{a \in R: a^{n}=0\right\}$ is a nilpotent $l$-ideal of index at most $n$.

Let $R$ be an $l$-algebra over $F$, and let $I$ be an $l$-ideal of $R$. Then $I_{1}=\{x \in R$ : $|x| \leqslant \alpha i$ for some $\alpha \in F^{+}$and $\left.i \in I^{+}\right\}$is the algebra $l$-ideal of $R$ generated by $I$. Since $I_{1}^{2} \subseteq I$, if $I$ is an $l$-prime $l$-ideal, then it is an algebra ideal. So $\beta(R)$ is the lower $l$-radical of the l-algebra $R$.

If $a$ is an element of the $l$-module $M$, then its positive part, negative part and absolute value are defined by $a^{+}=a \vee 0, a^{-}=(-a) \vee 0$ and $|a|=a \vee(-a)$, respectively. Then $a=a^{+}-a^{-},|a|=a^{+}+a^{-}$and $a^{+} \wedge a^{-}=0$. Moreover, if $a \wedge b=0$, then $a=x^{+}$and $b=x^{-}$for $x=a-b$. So for an $l$-ring $R(1)$ is equivalent to the 
identity $x^{+} y^{+} \wedge x^{-}=0$. Since $y^{+} x^{+} \wedge x^{-}=0$ is the corresponding identity for ${ }_{R} R$, the class of $f$-rings is a variety of $l$-rings. Also, each of the following conditions is equivalent to the corresponding parenthetical identity, and hence determines a variety of $l$-rings:

(3) $a \wedge b=0$ implies $a b=0\left(x^{+} x^{-}=0\right)$.

(4) $a^{2} \geqslant 0$ for each $a$ in $R\left(\left(x^{2}\right)^{-}=0\right)$.

The variety of $f$-rings is contained in the variety determined by (3); and the latter is contained in that determined by (4): $a^{2}=\left(a^{+}-a^{-}\right)^{2}=\left(a^{+}\right)^{2}+\left(a^{-}\right)^{2} \geqslant 0[4, \mathrm{p}$. 59]. Johnson [9, p. 174] has shown that an $l$-prime $f$-ring is a totally ordered domain (also see [10]), and Diem [5, p. 81] has shown that an $l$-prime $l$-ring which satisfies (3) is also a totally ordered domain (see Lemma 13 below).

Let $F[x, y]$ be a free noncommutative algebra over the totally ordered domain $F$. As a generalization of squares positive, a torsion-free $l$-algebra $R$ over $F$ is called a PPI l-algebra if there is a polynomial $f(x, y) \in F[x, y]$ such that $f(a, b) \geqslant 0$ for each $a, b \in R$ (we do not have any occasion to use more than two variables). Of course, we assume that $f(x, y) \notin F$, and if $R$ is not unital, then the constant term of $f(x, y)$ is zero. If for each $a$ in the $l$-algebra $R$ there is a polynomial $p(x)$ in $F[x]$ (of positive degree) with $p(a) \in R^{+}$, then $R$ will be called p-positive. A PPI l-algebra which satisfies $p(x) \geqslant 0$ is $p$-positive. In $\S 3$ we show that a unital $l$-prime $p$-positive $l$-algebra with properly conditioned polynomials is an $l$-domain, or even a domain.

In [12] Shyr and Viswanathan have called an $l$-ring $R$ square-archimedean if for each $a, b \in R^{+}$there is a positive integer $n$ such that $a b+b a \leqslant n\left(a^{2}+b^{2}\right)$. They showed that in a square archimedean $l$-ring $R, \beta(R)$ is the sum of the nilpotent $l$-ideals of $R$, and it is the largest nil $l$-ideal of $R$. In $\$ 3$ we consider polynomials more general than $f(x, y)=-(x y+y x)+n\left(x^{2}+y^{2}\right)$. We show that if $R$ is an $l$-prime $l$-algebra with the property that for some $a, b \in R^{+}$(or $a \in R$ ) there is a suitable polynomial $f(x, y)$ with $f(a, b) \geqslant 0$, then $R$ is an $l$-domain if it is unital, or satisfies more general conditions.

In $\S 4$ we summarize the results of $\S \S 2$ and 3 in terms of the lower $l$-radical $\beta(R)$ and strengthen the result of Shyr and Viswanathan. In $\$ 5$ we show that in an $l$-algebra with the polynomial constraints considered previously, if $a \wedge b=0$, then the $l$-ideals generated by $a b$ and $b a$ are identical. In $\$ 6$ there are some examples and a remark connecting the general constraints with (3) and (4).

Finally, we fix some notation and give a few more useful facts. If $X$ is a subset of the $l$-ring $R$, then $\langle X\rangle$ will denote the convex $l$-subgroup of $R$ generated by $X$. Also,

$$
M_{2}=\left\{a \in R^{+}: a^{2}=0\right\} .
$$

(5) If $R$ is a torsion free $l$-algebra over $F$ and $0<\beta \in F$ and $a \in R$ with $\beta a \geqslant 0$ $(\beta a \leqslant 0)$, then $a \geqslant 0(a \leqslant 0)$.

(6) $\left\langle R^{n}\right\rangle=\left\{r \in R:|r| \leqslant s^{n}\right.$ for some $\left.s \in R^{+}\right\}$is an $l$-ideal of $R$.

(7) If $a \wedge b=a \wedge c=0$, then $a \wedge(b+c)=0$.

(8) If $a, b \in R$ and $a_{1}=a-a \wedge b, b_{1}=b-a \wedge b$, then $a_{1} \wedge b_{1}=0$.

(9) If $a^{*} \wedge b^{*}=0$ in a homomorphic image $R^{*}$ of $R$, then there exist $a$ and $b$ in $R$, mapping to $a^{*}$ and $b^{*}$, respectively, and $a \wedge b=0$. 
2. Squares positive. Our first lemma is included for ease of reference, and is, for $F=\mathbf{Z}$ (except (d)), Example 15 of [4, p. 55]. The next two lemmas determine when an $l$-semiprime $l$-ring is $l$-reduced or reduced.

LEMMA 1. Let $R$ be a torsion-free l-algebra over the totally ordered domain $F$, and let

$$
T=\{c \in R:|c| \text { is an f-element of } R\} .
$$

Then:

(a) $T$ is a convex $f$-subalgebra of $R$.

(b) If $R$ is unital and $1>0$, then $F \subseteq T$.

(c) If $0 \neq \beta \in F$ and $a \in R$ with $\beta a \in T$, then $a \in T$.

(d) $R$ is a $T$ - $T$ f-bimodule.

Proof. We will only prove (c). If $x \wedge y=0$ in $R$, then $|\beta a| x \wedge y=0$ implies

$$
|\beta|(|a| x \wedge y)=|\beta||a| x \wedge|\beta| y=|\beta a| x \wedge|\beta| y=0 \text {. }
$$

So $|a| x \wedge y=0$ since $R$ is $F$-torsion-free; similarly, $x|a| \wedge y=0$, so $a \in T$.

We will consistently denote the f-subring of f-elements of $R$ by $T$, or $T(R)$, if necessary.

LEMMA 2. Let $R$ be an l-ring. If $a \in R^{+}$is an f-element of $R$ and $a^{2}=0$, then $a R a=0$.

Proof. Let $z \in R^{+}$. Then $(a z-z a)^{+} \wedge(a z-z a)^{-}=0$ and hence $(a z-z a)^{+} a$ $\wedge a(a z-z a)^{-}=0$. Since $(a z-z a)^{+} a=\left(a z a-z a^{2}\right)^{+}=a z a$ and $a(a z-z a)^{-}=$ $\left(a^{2} z-a z a\right)^{-}=a z a$, we have $a z a=a z a \wedge a z a=0$.

Recall that $M_{2}=\left\{a \in R^{+}: a^{2}=0\right\}$ and $N_{2}=\left\{a \in R: a^{2}=0\right\}$.

LeMma 3. Let $R$ be an l-ring.

(a) $R$ is l-reduced if and only if it is l-semiprime and $M_{2} \subseteq T$.

(b) $R$ is reduced if and only if it is l-semiprime and $N_{2} \subseteq T$.

(c) $R$ is an l-domain if and only if it is l-prime and $M_{2} \subseteq T$.

(d) $R$ is a reduced l-domain if and only if it is l-prime and $N_{2} \subseteq T$.

Proof. (a) If $R$ is $l$-semiprime and $M_{2} \subseteq T$, then $M_{2}=0$ by Lemma 2; hence $R$ is l-reduced.

(b) Suppose that $R$ is $l$-semiprime and $N_{2} \subseteq T$. If $a \in N_{2}$, then $|a| \in T$ and $|a|^{2}=\left|a^{2}\right|=0$ since $T$ is an $f$-subring. So $|a|=0$ by Lemma 2 , and hence $R$ is reduced.

(c) follows from (a), and (d) follows from (b).

In the following $T^{0}=\left\langle T^{0}\right\rangle$ is defined to be $\mathbf{Z}$ and $u^{0}=1$ (even if $1 \notin R$ ). The next result is a generalization of [14, Lemma 4(b), p. 203].

Lemma 4. Let $R$ be an l-ring with squares positive. Suppose that $a \in R$ and $k, l, m$, $n \in \mathbf{Z}^{+}$with $1 \leqslant l \leqslant m+k+2$. If $\left\langle T^{k}\right\rangle a^{2^{n}}\left\langle T^{m}\right\rangle \subseteq\left\langle T^{l}\right\rangle$, then

$$
\left\langle T^{k}\right\rangle a\left\langle T^{n+m}\right\rangle+\left\langle T^{k+n}\right\rangle a\left\langle T^{m}\right\rangle \subseteq\left\langle T^{l}\right\rangle .
$$

Proof. We use induction on $n$. If $n=0$ this is trivial. Suppose it is true for some integer $n$ and $\left\langle T^{k}\right\rangle a^{2^{n+1}}\left\langle T^{m}\right\rangle \subseteq\left\langle T^{l}\right\rangle$. Then $\left\langle T^{k}\right\rangle a^{2}\left\langle T^{n+m}\right\rangle+\left\langle T^{k+n}\right\rangle a^{2}\left\langle T^{m}\right\rangle \subseteq$ $\left\langle T^{l}\right\rangle$. If $t \in T^{+}$, then $0 \leqslant(a \pm t)^{2}$ yields $-\left(t^{2}+a^{2}\right) \leqslant t a+a t \leqslant t^{2}+a^{2}$ and 
hence $|t a+a t| \leqslant t^{2}+a^{2}$. But $R$ is a $T$ - $T f$-bimodule, and $|a t|,|t a| \leqslant|a t+t a|$ holds in any totally ordered $T$-T bimodule which is a homomorphic image of $R$, since $t \geqslant 0$; so it also holds in $R$. Now $|a t| \leqslant t^{2}+a^{2}$ implies

$$
\left|t^{k} a t^{n+m+1}\right|=t^{k}|a t| t^{n+m} \leqslant t^{k+n+m+2}+t^{k} a^{2} t^{n+m} \in\left\langle T^{l}\right\rangle
$$

so $t^{k} a t^{n+m+1} \in\left\langle T^{l}\right\rangle$. Thus $\left\langle T^{k}\right\rangle a\left\langle T^{n+m+1}\right\rangle \subseteq\left\langle T^{l}\right\rangle$ by (6), and, similarly, $\left\langle T^{k+n+1}\right\rangle a\left\langle T^{m}\right\rangle \subseteq\left\langle T^{l}\right\rangle$.

The subset $X$ of the $l$-ring $R$ is said to have local bi-f-superunits if for each $x \in X$ there is an element $e \in T^{+}$with $|x| \leqslant|x| e+e|x|+e|x| e$ (that is, $x$ is in the convex $l-T$ - $T$-bimodule of $R$ generated by $T x+x T$ ). The following theorem implies that a unital $l$-prime $l$-ring with squares positive is a domain.

THEOREM 1. Let $R$ be an l-ring in which the square of every element is positive.

(a) $R$ is l-reduced (an l-domain) if and only if it is l-semiprime (l-prime) and $M_{2}=\left\{a \in R^{+}: a^{2}=0\right\}$ has local bi-f-superunits.

(b) $R$ is reduced (a domain) if and only if it is l-semiprime (l-prime) and $N_{2}=\left\{a \in R: a^{2}=0\right\}$ has local bi-f-superunits.

Proof. (a) Suppose that $R$ is $l$-semiprime and $M_{2}$ has local bi-f-superunits. If $a \in M_{2}$, then by Lemma 4, with $k=m=0$ and $n=l=1, a T+T a \subseteq T$, and hence $a T+T a+T a T \subseteq T$. If $U$ is the convex $l$-subgroup of $R$ generated by $a T+T a+T a T$, then $U=\left\{u \in R:|u| \leqslant a t+t a+t a t\right.$ for some $\left.t \in T^{+}\right\} \subseteq T$, and $a \in U$ since $a$ has a bi-f-superunit. So $M_{2} \subseteq T$ and $R$ is $l$-reduced by Lemma 3(a). If $R$ is also $l$-prime, then it is an $l$-domain by Lemma 3(c).

(b) If $R$ is $l$-semiprime and $N_{2}$ has local bi-f-superunits, then, as in the previous paragraph, $N_{2} \subseteq T$. So $R$ is reduced by Lemma 3(b). If $R$ is also l-prime, then it is a reduced $l$-domain. But if $a b=0$, then $a^{2} b^{2}=0$ implies $a^{2}=0$ or $b^{2}=0$, and hence $a=0$ or $b=0$.

Another version of Theorem 1 is implied by the following two lemmas. The left annihilator of a subset $X$ of $R$ is $l_{R}(X)=\{a \in R: a x=0$ for each $x \in X\}$; the right annihilator of $X$ will be denoted by $r_{R}(X)$.

Lemma 5. Let $R$ be an l-ring and suppose that $X \subseteq T$ with $X \subseteq X_{1}-X_{1}$ where $X_{1}=\left(X \cap R^{+}\right) \cup\{0\}$. Then $r_{R}(X)=r_{R}(\langle X\rangle)$ is a right l-ideal of $R$, and $l_{R}(X)=$ $l_{R}(\langle X\rangle)$ is a left l-ideal of $R$.

Proof. Let $x \in X$ and $r \in r_{R}(X)$. Then $x=x_{1}-x_{2}$ where $x_{1}, x_{2} \geqslant 0$ and $x_{1}$, $x_{2} \in X \cup\{0\}$. If $|s| \leqslant|r|$, then

$$
\begin{aligned}
|x s| & =\left|\left(x_{1}-x_{2}\right) s\right| \leqslant\left|x_{1} s\right|+\left|x_{2} s\right| \\
& =x_{1}|s|+x_{2}|s| \leqslant x_{1}|r|+x_{2}|r|=\left|x_{1} r\right|+\left|x_{2} r\right|=0 .
\end{aligned}
$$

So $s \in r_{R}(X)$ and $r_{R}(X)$ is a right $l$-ideal of $R$. Since $X \subseteq\langle X\rangle, r_{R}(\langle X\rangle) \subseteq r_{R}(X)$. Since $\langle X\rangle=\left\{u \in R:|u| \leqslant x_{1}+\cdots+x_{n}\right.$ for some $\left.0 \leqslant x_{i} \in X_{1}\right\}$, if $r \in r_{R}(X)$ and $u \in\langle X\rangle$ with $|u| \leqslant x_{1}+\cdots+x_{n}$, then $|u r| \leqslant|u||r| \leqslant x_{1}|r|+\cdots+x_{n}|r|=0$, since $|r| \in r_{R}(X)$. Thus $u r=0$ and $r \in r_{R}(\langle X\rangle)$. So $r_{R}(X) \subseteq r_{R}(\langle X\rangle)$. Similarly, $l_{R}(X)=l_{R}(\langle X\rangle)$ is a left $l$-ideal of $R$. 
LEMMA 6. Let $R$ be an l-ring with squares positive and suppose that $a \in R$ with $a^{2^{n}} \in T$. If $u \wedge v=0$ in $R$, then $|a| u \wedge v \in r_{R}\left(T^{n}\right)$ and $u|a| \wedge v \in l_{R}\left(T^{n}\right)$. (If $n=0, r_{R}\left(T^{n}\right)=l_{R}\left(T^{n}\right)=0$.)

Proof. By Lemma 4 with $k=m=0$ and $l=1, a T^{n}+T^{n} a \subseteq T$. If $n=0$ the result is obvious; so assume $n \geqslant 1$. If $0 \leqslant s \in\left\langle T^{n}\right\rangle$, then $s \leqslant t^{n}$ for some $t \in T^{+}$by (6). So $s(|a| u \wedge v) \leqslant t^{n}(|a| u \wedge v)=\left|t^{n} a\right| u \wedge t^{n} v=0$. Since $\left\langle T^{n}\right\rangle=\left\langle T^{n}\right\rangle^{+}$ $-\left\langle T^{n}\right\rangle^{+},|a| u \wedge v \in r_{R}\left(\left\langle T^{n}\right\rangle\right)=r_{R}\left(T^{n}\right)$ by Lemma 5 .

THEOREM 2. Let $R$ be an l-ring in which the square of every element is positive and suppose that $l_{R}(T)=r_{R}(T)=0$. Then:

(a) $R$ is reduced if and only if it is l-semiprime.

(b) $R$ is a domain if and only if it is l-prime.

Proof. By Lemma 6, $N_{2} \subseteq T$, and hence (a) follows from Lemma 3(b). If $R$ is $l$-prime, then it is a reduced $l$-domain by Lemma $3(\mathrm{~d})$, and hence a domain (see the proof of Theorem 1).

3. Polynomial constraints which generalize squares positive. In this section we show that Theorems 1 and 2 are true for $l$-algebras which satisfy polynomial constraints more general than $x^{2} \geqslant 0$. The types of constraints that we use are illustrated in the next two results which are generalizations of [14, Theorem 7, p. 200].

Let $F$ be a totally ordered domain. A polynomial $f(x, y) \in F[x, y]$ will be called nice if it has at least one monomial of degree 1 in $x$ and each of its monomials of degree 1 in $x$ has a negative coefficient. So if $f(x, y)$ is nice, then $f(x, y)=-g(x, y)$ $+p(y)+h(x, y)$ where $0 \neq g(x, y)$ is of degree 1 in $x$ and all its coefficients are positive, and $h(x, y)=0$ or each of its monomials is of degree at least 2 in $x$. For example, for each $\alpha \in F, f(x, y)=-(x y+y x)+\alpha\left(x^{2}+y^{2}\right)$ is nice; so is $(y-x)^{n}$ and modifications obtained by putting in appropriate coefficients $\alpha \in F$ in the monomials of $(y-x)^{n}$. Note that $y$ need not appear in the nice polynomial $f(x, y)$. We will consistently denote the "parts" of a nice polynomial $f(x, y)$ by $g(x, y), p(y)$ and $h(x, y)$, as in the definition.

The derivative of $p(x) \in F[x]$ will be denoted by $p^{\prime}(x)$. If $f(x, y)$ is a nice polynomial then $f(x, 1)^{\prime}(0)<0$.

LEMMA 7. Let $R$ be a unital torsion-free l-algebra over the totally ordered domain $F$. The following statements are equivalent for the nilpotent element a of $R$.

(a) $|a|<1$.

(b) There is a polynomial $p(x)$ in $F\left[x^{2}\right]$ with $p\left(a^{n}+1\right) \geqslant 0$ and $p\left(a^{n}-1\right) \geqslant 0$ for each $n \geqslant 1$, and $0 \neq p^{\prime}(1) \cdot 1 \in R^{+}$.

(c) For each integer $n \geqslant 1$ there are polynomials $p_{1}(x)$ and $q_{1}(x)$ in $F[x]$ with $p_{1}\left(a^{n}+1\right) \geqslant 0, q_{1}\left(\left(a^{n}-1\right)^{2}\right) \geqslant 0$ and $p_{1}^{\prime}(1) q_{1}^{\prime}(1) \cdot 1>0$ in $R$.

(d) For each integer $n \geqslant 1$ there are polynomials $p_{2}(x)$ and $q_{2}(x)$ in $F[x]$ with $p_{2}\left(a^{n}+1\right) \geqslant 0, q_{2}\left(a^{n}-1\right) \geqslant 0$ and $p_{2}^{\prime}(1) q_{2}^{\prime}(-1) \cdot 1<0$ in $R$.

(e) $1 \in R^{+}$and for each $b$ in $\left\{ \pm a^{n}: n \geqslant 1\right\}$ there is a polynomial $f(x, y) \in F[x, y]$ such that $f(b, 1) \geqslant 0$ and $f(x, 1)^{\prime}(0)<0$. 
(f) $1 \in R^{+},|a|$ is nilpotent and if $u \wedge v=0$ with $u \leqslant\left|a^{m}\right|$ for some $m \in \mathbf{Z}^{+}$and $v \leqslant 1$, then there is a nice polynomial $f(x, y) \in F[x, y]$ with $f(u, v) \geqslant 0$.

(g) For each integer $n \geqslant 1$ there are polynomials $p_{3}(x)$ and $q_{3}(x) \in F[x]$, with only odd terms, such that $p_{3}(b)^{+} p_{3}(b)^{-}=0$ if $b= \pm\left(a^{n}+1\right)$, and $q_{3}(b)^{+} q_{3}(b)^{-}=0$ if $b= \pm\left(a^{n}-1\right)$; and $p_{3}(1) p_{3}^{\prime}(1) q_{3}(1) q_{3}^{\prime}(1) \cdot 1>0$ in $R$.

Proof. For (a) $\rightarrow$ (b) let $p(x)=x^{2}$ and use the fact that $T$ is an $f$-ring (Lemma 1(a)). For (b) $\rightarrow$ (c) let $p_{1}(x)=p(x)$ and $q_{1}(x)=h(x)$ where $p(x)=h\left(x^{2}\right)$ in (b). For (c) $\rightarrow$ (d) let $q_{2}(x)=q_{1}\left(x^{2}\right)$ and $p_{2}(x)=p_{1}(x)$.

$(\mathrm{d}) \rightarrow(\mathrm{e})$. Let $b=a^{n}$ and take $p_{2}(x), q_{2}(x) \in F[x]$ with $p_{2}\left(a^{n}+1\right) \geqslant 0$, $q_{2}\left(a^{n}-1\right) \geqslant 0$ and $p_{2}^{\prime}(1) q_{2}^{\prime}(-1) \cdot 1<0$. If $\beta=p_{2}^{\prime}(1) q_{2}^{\prime}(-1)>0$, then $1<0$ in $R$ by (5). So $\beta<0,(-\beta) \cdot 1>0$ and $1 \in R^{+}$. Now

$$
\begin{aligned}
0 & \leqslant q_{2}(b-1)=\alpha_{0}+\alpha_{1}(b-1)+\alpha_{2}(b-1)^{2}+\cdots+\alpha_{m}(b-1)^{m} \\
& =\left(\alpha_{1}-2 \alpha_{2}+\cdots+(-1)^{m-1} m \alpha_{m}\right) b+\alpha_{0}+h(b) \\
& =q_{2}^{\prime}(-1) b+\alpha_{0}+h(b)
\end{aligned}
$$

where $h(x) \in x^{2} F[x]$. Similarly, there exists $h_{1}(x) \in x^{2} F[x]$ with

$$
0 \leqslant p_{2}(b+1)=p_{2}^{\prime}(1) b+\gamma_{0}+h_{1}(b) \text {. }
$$

If $q_{2}^{\prime}(-1)<0$, then $f_{+}(x, y)=q_{2}^{\prime}(-1) x+\alpha_{0}+h(x)$ is a nice polynomial with $f_{+}(b, 1) \geqslant 0$. Also, $p_{2}^{\prime}(1)>0$ since $p_{2}^{\prime}(1) q_{2}^{\prime}(-1)<0$, and $f_{-}(x, y)=-p_{2}^{\prime}(1) x+\gamma_{0}+$ $h_{2}(x)$ is a nice polynomial with $f_{-}(-b, 1) \geqslant 0$; here, if $h_{1}(x)=\Sigma \gamma_{i} x^{i}$, then $h_{2}(x)=$ $\Sigma(-1)^{i} \gamma_{i} x^{i}$

If $q_{2}^{\prime}(-1)>0$, then again we get two nice polynomials $f_{ \pm}(x, y)$ with $f_{+}(b, 1) \geqslant 0$ and $f_{-}(-b, 1) \geqslant 0$.

(e) $\rightarrow$ (a). By induction on the index of nilpotency of $a$ we may assume that $a^{k} \in T$ if $k \geqslant 2$. Let $f(x, y)=g(x, y)+p(y)+h(x, y)$ be a polynomial with $f(x, 1)^{\prime}(0)<0$ and $f(a, 1)=g(a, 1)+p(1)+h(a, 1) \geqslant 0$, where the monomials of $g(x, y)$ (respectively, $h(x, y))$ are of degree 1 (respectively, 2) in $x$. Then, since $g(a, 1)=-\beta a$ where $\beta=-f(x, 1)^{\prime}(0)>0$ and $h(a, 1) \in a^{2} F[a] \subseteq T$, we have $\beta a \leqslant s$ for some $s \in T$. By using a similar polynomial for $-a$, we get $-\gamma a \leqslant t$ for some $t \in T$ and $0<\gamma \in F$. So $-\beta t \leqslant \gamma \beta a \leqslant \gamma s$ and $a \in T$ by Lemma 1(a) and (c). Since (a) holds in any totally ordered ring, it must hold in any $f$-ring.

(f) $\rightarrow$ (a). By induction on the index of nilpotency of $b=|a|$, we may assume that $b^{n}=0, n \geqslant 2$, and $b^{k} \in T$ if $k \geqslant 2$. Let $c=b \wedge 1$, and let $u=b-c$ and $v=1-c$. Then $c, v \in T$ and $u \wedge v=0$ by (8). Let $f(x, y)=-g(x, y)+p(y)+h(x, y)$ be a nice polynomial with $f(u, v) \geqslant 0$. Then $0 \leqslant g(u, v) \leqslant p(v)+h(u, v)$. Each term of $h(u, v)$ is of the form $\alpha w=\alpha u^{n_{1}} v^{m_{1}} u^{n_{2}} v^{m_{2}} \cdots u^{n_{t}} v^{m_{t}}$ with $N=\sum n_{i} \geqslant 2$. Since $v \leqslant 1,0 \leqslant w \leqslant u^{N} \leqslant b^{N} \in T$; so $\alpha w \in T$ and hence $h(u, v) \in T$. Whence $g(u, v) \in$ $T$ since $p(v) \in T$. Now $g(u, v)$ contains a term of the form $\alpha u, \alpha u v^{m}, \alpha v^{m} u$ or $\alpha v^{m} u v^{k}$, where $\alpha>0$ and $m, k \geqslant 0$. Since $g(x, y)$ has positive coefficients, if $d$ is this term, then $0 \leqslant d \leqslant g(u, v)$ and hence $u, u v^{m}, v^{m} u$ or $v^{m} u v^{k} \in T$ (Lemma $\left.1(\mathrm{c})\right)$. But $v=1-c$ is an invertible element in $T$ (since $c^{n}=0$ ), hence $u \in T$ and $b=u+c \in T$. 
$(\mathrm{g}) \rightarrow(\mathrm{d})$. Since $p_{3}(x)$ has only odd terms $p_{3}(-b)=-p_{3}(b)$; and hence $p_{3}(-b)^{+}=$ $p_{3}(b)^{-}$and $p_{3}(-b)^{-}=p_{3}(b)^{+}$. So if $b=a^{n}+1$, then $p_{3}(b)^{+} p_{3}(b)^{-}=0$ and $p_{3}(b)^{-} p_{3}(b)^{+}=0$, and hence

$$
p_{3}(b)^{2}=\left[p_{3}(b)^{+}-p_{3}(b)^{-}\right]^{2}=\left[p_{3}(b)^{+}\right]^{2}+\left[p_{3}(b)^{-}\right]^{2} \geqslant 0
$$

Similarly, $q_{3}(b)^{2} \geqslant 0$ if $b=a^{n}-1$. Let $p_{2}(x)=p_{3}(x)^{2}$ and $q_{2}(x)=q_{3}(x)^{2}$. Then $p_{2}\left(a^{n}+1\right) \geqslant 0, q_{2}\left(a^{n}-1\right) \geqslant 0$ and $p_{2}^{\prime}(1) q_{2}^{\prime}(-1) \cdot 1<0$ in $R$.

Since $T$ is a convex $f$-subring of $R$ (Lemma l(a)) and hence satisfies (3) and (4), for the implication (a) $\rightarrow$ (f) we may let $f(x, y)=-(x y+y x)+x^{2}+y^{2}$, and for (a) $\rightarrow(\mathrm{g})$ we may let $p_{3}(x)=q_{3}(x)=x$. The proof is complete.

The next lemma shows that polynomials also determine when the idempotents are in $T$.

LEMMA 8. The following statements are equivalent for the unital torsion-free l-algebra $R$ over the totally ordered domain $F$.

(a) The idempotents of $R$ are contained in the interval $[0,1]$ (and are central).

(b) There is a polynomial $p(x)$ in $F[x]$ with $p(f) \geqslant 0$ for each idempotent $f$, and [ $p(1)-p(0)] \cdot 1>0$ in $R$.

(c) For each idempotent $f$ there are polynomials $p(x)$ and $q(x)$ in $F[x]$ with $p(f) \geqslant 0, q(1-f) \geqslant 0$ and $[p(1)-p(0)][q(1)-q(0)] \cdot 1>0$ in $R$.

(d) For each idempotent $f$ there are polynomials $p(x)$ and $q(x)$ in $F[x]$, with zero constant terms, such that $p(f)^{+} p(f)^{-}=q(f)^{-} q(f)^{+}=0$ and $p(1) q(1)>0$.

Proof. Since $T$ is an $f$-ring (Lemma l(a)) squares are positive in $T$ and $T$ satisfies $x^{+} x^{-}=0$; so (a) implies (b) and (d), and clearly (b) implies (c). Also, for (d) implies (a) we can simply note that for $f$ idempotent $p(f)=p(1) f$ and $q(f)=q(1) f$, and so $f^{+} f^{-}=f^{-} f^{+}=0$. Hence $f=f^{2} \geqslant 0$ and $1-f \geqslant 0$. Now we show that (c) $\rightarrow(\mathrm{a})$.

By (5) $1 \in R^{+}$, since $[p(1)-p(0)][q(1)-q(0)] \cdot 1>0$. Also $0 \leqslant p(f)=p(0)+$ $[p(1)-p(0)] f$ and $0 \leqslant q(1-f)=q(1)-[q(1)-q(0)] f$ yield

$$
-p(0) \leqslant[p(1)-p(0)] f \text { and }[q(1)-q(0)] f \leqslant q(1) \text {. }
$$

So, as in the proof of (e) $\rightarrow$ (a) of Lemma 7, $f \in T$. But (a) is satisfied in any unital $f$-algebra [7, p. 539]. For if $f=f^{2}$ in a unital totally ordered algebra, then $0 \leqslant f \leqslant 1$ $-f$ or $0 \leqslant 1-f \leqslant f$, and hence $f=0$ or 1 . Thus, a unital $f$-algebra satisfies (a), since it is a subdirect product of totally ordered algebras. Consequently, by Lemma 1(a), the idempotents of $R$ are contained in $[0,1]$ and commute, and hence are central.

Note that the conditions on the coefficients of the polynomials are important. For any algebraic $l$-algebra $R$ will satisfy the constraint $p(a) \in R^{+}$, but it need not satisfy (a) of Lemmas 7 and 8.

Results analogous to Theorem 1 follow from Lemmas 7 and 3. We state one such result which uses (d) of Lemma 7. 
THEOREM 3. Let $R$ be a unital torsion-free l-algebra over the totally ordered domain $F$.

(a) $R$ is l-reduced (an l-domain) with $1 \in R^{+}$if and only if $R$ is l-semiprime (l-prime) and for each element $a$ in $M_{2}=\left\{a \in R^{+}: a^{2}=0\right\}$ there is a polynomial $q_{2}(x)$ in $F[x]$ with $q_{2}(a-1) \geqslant 0$ and $q_{2}^{\prime}(-1) \cdot 1<0$ in $R$.

(b) $R$ is reduced (a reduced l-domain) with $1 \in R^{+}$if and only if $R$ is l-semiprime (l-prime) and for each element $a$ in $N_{2}=\left\{a \in R: a^{2}=0\right\}$ there are polynomials $p_{2}(x)$ and $q_{2}(x)$ in $F[x]$ with $p_{2}(a+1) \geqslant 0, q_{2}(a-1) \geqslant 0$ and $p_{2}^{\prime}(1) q_{2}^{\prime}(-1) \cdot 1<0$ in $R$.

Next, we determine, in terms of polynomial constraints, when a unital $l$-domain is a domain. Let $\bar{F}$ be the totally ordered field of quotients of the totally ordered domain $F$, and let $R$ be a torsion-free $l$-algebra over $F$. Then $\bar{R}=R \otimes_{F} \bar{F}=\{r / \alpha$ : $r \in R$ and $0 \neq \alpha \in F\}$ is the $F$-divisible hull of $R$. If $\bar{R}$ is given the positive cone $\bar{R}^{+}=\left\{r / \alpha: r \in R^{+}\right.$and $\left.\alpha \in F^{+}\right\}$, then $\bar{R}$ is an $l$-algebra over $\bar{F}$ which contains $R$.

The $F$-l-algebra $R$ will be called normal (i-normal) if for each $a$ in $R$ which is a zero divisor there is a polynomial $0 \neq p(x)$ in $F[x]$, with zero constant term, such that $p(a) \geqslant 0$ (and $p(1) \neq 0)$.

LEMMA 9. Let $R$ be a unital, reduced, normal l-algebra over the totally ordered domain $F$, and suppose that $R$ is an l-domain. Then the following statements are equivalent.

(a) $R$ is a domain and $1 \in R^{+}$.

(b) If $c^{2}=\alpha c$ with $c \in R$ and $0<\alpha \in F$, then there is a polynomial $p(x)$ in $F[x]$ such that $p(c) \in R^{+}$and $[p(\alpha)-p(0)] \cdot 1>0$ in $R$.

(c) The idempotents of $\bar{R}=R \otimes_{F} \bar{F}$ are positive.

(d) $\bar{R}$ is i-normal over $\bar{F}$ and $1 \in R^{+}$.

Proof. (a) $\rightarrow$ (b). If $c^{2}=\alpha c$ with $\alpha>0$, then $f=c / \alpha$ is an idempotent of $\bar{R}$, and since $\bar{R}$ is a domain, $f=0$ or 1 . So $c=0$ or $\alpha$ and we can let $p(x)=x$.

(b) $\rightarrow$ (c). First note that $1 \in R^{+}$by (5). Let $f=c / \alpha$ be an idempotent in $\bar{R}$ with $\alpha>0$. Then $1-f=(\alpha-c) / \alpha$ is idempotent and $c^{2}=\alpha c$ and $(\alpha-c)^{2}=\alpha(\alpha-c)$. Let $p(x), q(x) \in F[x]$ be such that $p(c) \geqslant 0, q(\alpha-c) \geqslant 0$ and $p(\alpha)-p(0)>0$, $q(\alpha)-q(0)>0$. Then $p(c)=p(\alpha f)=p(0)+[p(\alpha)-p(0)] f \geqslant 0$ and $q(\alpha-c)=$ $q(\alpha(1-f))=q(0)+[q(\alpha)-q(0)](1-f) \geqslant 0$. So $-p(0) \leqslant[p(\alpha)-p(0)] f$ and $[q(\alpha)-q(0)] f \leqslant q(\alpha)$, and hence $f \in T(\bar{R})$ since $F \subseteq T$ by Lemma 1 .

(c) $\rightarrow$ (a). Since $\bar{T}=T \otimes_{F} \bar{F}$ is the set of $f$-elements of the $l$-domain $\bar{R}, \bar{T}$ is an $f$-ring (Lemma $1(\mathrm{a})$ ) and hence is a domain. But the idempotents of $\bar{R}$, being positive, are contained in $\bar{T}$; and hence 0 and 1 are the only idempotents of $\bar{R}$. Let $a b=0$ in $R$; then, since $R$ is a normal $l$-algebra, there are nonzero polynomials $p(x)$ and $q(x)$ in $x F[x]$ with $p(a) \geqslant 0$ and $q(b) \geqslant 0$. Since $R$ is an $l$-domain and $p(a) q(b)=0$, either $p(a)=0$ or $q(b)=0$; suppose $p(a)=0$ and $a \neq 0$. Then, since $\bar{R}$ is reduced, the algebraic element $a$ is strongly regular in $\bar{F}[a]$; that is, $a=a^{2} h(a)$ for some polynomial $h(x)$ in $\bar{F}[x]$. For, since $\bar{F}[a]$ is reduced, $\bar{F}[a] \simeq$ $\bar{F}[x] /(g(x))$ with $g(x)$ square free; so that $\bar{F}[a]$, as a ring, is a direct sum of fields (or see [8, p. 165]). Since $f=a h(a)$ is an idempotent of $\bar{R}, f=0$ or $f=1$; thus $f=1$ and $b=0$. 
(d) $\rightarrow$ (c). Let $f \neq 0,1$ be an idempotent of $\bar{R}$. Since $\bar{R}$ is $i$-normal there exists $p(x) \in x \bar{F}[x]$ with $0 \leqslant p(f)=p(1) f$ and $p(1) \neq 0$. Then $p(1)^{2} f \geqslant 0$ and hence $f \geqslant 0$ by (5).

Since (a) trivially implies (d) the proof is complete.

Note that the equivalence of (b) and (c) in Lemma 9 holds for any unital $l$-algebra. From Theorem 3 and Lemmas 7 and 9 we get the following two corollaries.

COROLlaRY 1. Let $R$ be a unital torsion-free l-algebra over the totally ordered domain $F$. Then $R$ is a domain with $1 \in R^{+}$if and only if it is a normal l-prime l-algebra which satisfies (i) and (ii).

(i) If $a \in R$ with $a^{2}=0$, then there are polynomials $p_{2}(x)$ and $q_{2}(x) \in F[x]$ with $p_{2}(a+1) \geqslant 0, q_{2}(a-1) \geqslant 0$ and $p_{2}^{\prime}(1) q_{2}^{\prime}(-1) \cdot 1<0$ in $R$.

(ii) If $c^{2}=\alpha c$ where $0<\alpha \in F$ and $c \in R$, then there exists $p(x) \in F[x]$ with $p(c) \in R^{+}$and $[p(\alpha)-p(0)]>0$.

The $F$-l-algebra is weakly p-positive if for each $a$ in $R$ there is a polynomial $p(x) \in F[x]$ (of degree $\geqslant 1$ ) with $p(a) \geqslant 0$ and $p^{\prime}(1)>0$ in $F$; it is strongly $p$-positive if for each $a$ in $R, p(x)$ exists with positive coefficients with $p(a) \geqslant 0$.

COROLlaRy 2. Let $R$ be a unital, weakly p-positve, torsion-free l-algebra over the totally ordered domain $F$.

(a) If $1 \in R^{+}$, then $R$ is a reduced l-domain if and only if it is l-prime.

(b) If $F$ is a field and $1 \in R^{+}$, then $R$ is a domain if and only if it is an i-normal l-prime l-algebra.

(c) If $R$ is strongly p-positive, then $1 \in R^{+}$and $R$ is a domain if and only if it is a normal l-prime l-algebra.

Proof. (a) follows from Lemmas 7(c) and 3(d), and then (b) follows from Lemma 9(d). If $R$ is a strongly $p$-positive normal $l$-prime $l$-algebra, then $p(1) \cdot 1 \in R^{+}$with $p(x) \in F^{+}[x]$ implies $1 \in R^{+}$, and hence $F^{+} \subseteq R^{+}$. Thus $R$ is a domain by Corollary 1.

Example 1 in $\S 6$ shows that (b) is false if $F=\mathbf{Z}$, even if $R$ is commutative and the idempotents of $R$ are positive. It also shows that a weakly $p$-positive $l$-algebra need not be strongly $p$-positive. We also note that [16, Example 2] shows that a commutative unital $l$-domain with all idempotents positive, which is a $p$-positive $l$-algebra over a totally ordered field $F$, need not be reduced. In this example each element $a$ satisfies an inequality $(x-\alpha)^{2} \geqslant 0$. In fact, if $R$ is any $l$-algebra with squares positive and $R_{1}=R+F$ is the $l$-algebra obtained from $R$ by freely adjoining $F$ in the usual manner (so $R_{1}^{+}=\left\{(r, \alpha): r \in R^{+}\right.$and $\left.\alpha \in F^{+}\right\}$), then $R_{1}$ is a $p$-positive l-algebra with $1>0$. Each element of $R_{1}$ satisfies $(x-\alpha)^{2} \geqslant 0$ for some $\alpha \in F$. $R_{1}$ will be an $l$-domain if $R$ is an $l$-domain. Analogous statements are true for any $p$-positive $l$-algebra.

If $A$ is a finite subset of a strongly $p$-positive $l$-algebra $R$, then there is a polynomial $p(x) \in F^{+}[x]$ with $p(a) \geqslant 0$ for each $a$ in $A$. For if $a_{1}$ and $a_{2}$ are in $R$ and if $p_{1}(x), p_{2}(x) \in F^{+}[x]$ with $p_{2}\left(a_{2}\right) \in R^{+}$and $p_{1}\left(p_{2}\left(a_{1}\right)\right) \in R^{+}$, then $p\left(a_{i}\right) \in$ $R^{+}$for $i=1,2$ where $p(x)=p_{1}\left(p_{2}(x)\right)$. Similarly, the direct sum of a family of 
strongly $p$-positive $l$-algebras is strongly $p$-positive. Since the direct sum need not be unital, we note that throughout this paper, the condition " $1 \in R^{+}$" may be replaced by " $R$ has central $f$-units"; that is, for each $a \in R$ there is an idempotent $e$ in $T$ which is central in $R$ and $a=a e$.

We turn next to two-variable polynomials and give the following generalization of Lemma 4.

LEMMA 10. Let $R$ be a torsion-free l-algebra over the totally ordered domain $F$. Suppose that $a \in R$ and $1 \leqslant k \in \mathbf{Z}$. Assume that for each $t \in T^{+}$and each integer $m \geqslant 0$ there are two nice polynomials $f_{i}(x, y)=-g_{i}(x, y)+p_{i}(y)+h_{i}(x, y) \in$ $F[x, y], i=1,2$, with $f_{1}\left(a^{k^{m}}, t\right) \geqslant 0, f_{2}\left(-a^{k^{m}}, t\right) \geqslant 0$ and such that:

(i) $g_{1}(x, y)$ or $g_{2}(x, y)$ has a monomial ending in $x$ and $g_{2}\left(a^{k^{m}}, t\right) \leqslant g_{1}\left(a^{k^{m}}, t\right)$.

(ii) $h_{i}(x, y) \in F\left[x^{k}, y\right]$; so $h_{i}(x, y)=q_{i}\left(x^{k}, y\right)$ for $i=1,2$.

If $a^{k^{n}} \in T$ for some $n \geqslant 0$, then for each $s \in T \cup\{1\}$ and for each $t \in T$ there is an integer $N \geqslant 0$ with $t^{N}$ sa $\in T$.

Moreover, if the degree in $y$ of each monomial of $g_{i}(x, y)$ which ends in $x$ (for all $t \in T^{+}$and $\left.m \geqslant 0\right)$ is bounded by $M_{1}$, and the degree of each $q_{i}(x, y)$ in $x$ is bounded by $M_{2}$, then we may take $N \leqslant M_{1}\left(M_{2}^{n}+M_{2}^{n-1}+\cdots+1\right)$.

Proof. Let $t \in T$ and $s \in T \cup\{1\}$. We may assume that $s \geqslant 0$ and $t \geqslant 0$. For if $|t|^{N}|s| a \in T$, then

$$
\left|t^{N} s a\right| \leqslant|t|^{N}|s||a|=\left.|| t\right|^{N}|s| a \mid \in T
$$

implies that $t^{N} s a \in T$ by Lemma 1 . Let $t_{1}=t \vee s$ if $s \neq 1$ and let $t_{1}=t$ if $s=1$. We argue by induction on $n$. If $n=0$, then $a \in T$ and we can let $N=0$. Assume the result is true for the integer $n$ and $a^{k^{n+1}} \in T$, and let $b=a^{k}$. Then $b^{k^{n}} \in T$ and hence for each $s_{1} \in T \cup\{1\}$ there is an integer $N_{1}$ with $t_{1}^{N_{1}} s_{1} b \in T$ (and $N_{1} \leqslant M_{1}\left(M_{2}^{n}+M_{2}^{n-1}+\cdots+1\right)$ if $M_{1}$ and $M_{2}$ exist). Now for each integer $r \geqslant 1$ there is an integer $N_{r}$ with $t_{1}^{N_{r}} s_{1} b^{r} \in T$ (and $N_{r} \leqslant r M_{1}\left(M_{2}^{n}+M_{2}^{n-1}\right.$ $+\cdots+1)$ ). For if $s_{2}=t_{1}^{N_{r}} s_{1} b^{r} \in T$, then there exists an integer $M$ with $t_{1}^{M} s_{2} b \in T$ (and $M \leqslant M_{1}\left(M_{2}^{n}+M_{2}^{n-1}+\cdots+1\right)$ ); but $t_{1}^{M} s_{2} b=t_{1}^{M} t_{1}^{N_{r}} s_{1} b^{r+1}$ and hence $N_{r+1}=$ $M+N_{r}\left(\right.$ and $\left.N_{r+1} \leqslant(r+1) M_{1}\left(M_{2}^{n}+M_{2}^{n-1}+\cdots+1\right)\right)$.

Let $f_{1}(x, y)=-g_{1}(x, y)+p_{1}(y)+h_{1}(x, y)$ be a nice polynomial which satisfies (ii) and such that $f_{1}\left(a, t_{1}\right) \geqslant 0$. If $u$ is a term of $h_{1}\left(a, t_{1}\right)=q_{1}\left(a^{k}, t_{1}\right)=q_{1}\left(b, t_{1}\right)$, then

$$
u=\alpha t_{1}^{i_{1}} b^{j_{1}} t_{1}^{i_{2}} b^{j_{2}} \cdots t_{1}^{i_{1}} b^{j_{1}}
$$

with $0 \neq \alpha \in F, l \geqslant 1, i_{1} \geqslant 0, j_{l} \geqslant 0$ and $j_{1} \geqslant 1$. We claim that $t_{1}^{L} u \in T$ for some $L$ (and $L \leqslant\left(\sum_{\nu=1}^{l} j_{\nu}\right) M_{1}\left(M_{2}^{n}+M_{2}^{n-1}+\cdots+1\right)$ ). If $l=1$ this follows from the previous paragraph. Assume that $l \geqslant 2$ and $t_{1}^{L_{1}}\left(\alpha t_{1}^{i_{1}} b^{j_{1}} \cdots t_{1}^{i_{l-1}} b^{j_{l-1}}\right)=s_{3} \in T$ (and $L_{1} \leqslant$ $\left.\left(\sum_{\nu=1}^{l-1} j_{\nu}\right) M_{1}\left(M_{2}^{n}+M_{2}^{n-1}+\cdots+1\right)\right)$. Then, again, there is an integer $L_{2}$ with

$$
t_{1}^{L_{1}+L_{2}} u=t_{1}^{L_{2}}\left(s_{3} t_{1}^{i_{l}}\right) b^{j_{l}} \in T
$$

and so

$$
L=L_{1}+L_{2}
$$


(and

$$
\left.L \leqslant\left(\sum_{\nu=1}^{l} j_{\nu}\right) M_{1}\left(M_{2}^{n}+M_{2}^{n-1}+\cdots+1\right) \leqslant M_{1}\left(M_{2}^{n+1}+M_{2}^{n}+\cdots+M_{2}\right)\right) .
$$

Thus, there exists $L_{3}$ with $t_{1}^{L_{3}} h_{1}\left(a, t_{1}\right) \in T$ (and $L_{3} \leqslant M_{1}\left(M_{2}^{n+1}+\cdots+M_{2}\right)$ ).

Similarly, if $f_{2}(x, y)=-g_{2}(x, y)+p_{2}(y)+h_{2}(x, y)$ is a nice polynomial which satisfies (i) and (ii) and $f_{2}\left(-a, t_{1}\right) \geqslant 0$, then there is an integer $L_{4}$ with $t_{1}^{L_{4}} h_{2}\left(-a, t_{1}\right)$ $\in T$ (and $L_{4} \leqslant M_{1}\left(M_{2}^{n+1}+M_{2}^{n}+\cdots+M_{2}\right)$ ). Let $L_{5}$ be the larger of $L_{3}$ and $L_{4}$ $\left(L_{5} \leqslant M_{1}\left(M_{2}^{n+1}+M_{2}^{n}+\cdots+M_{2}\right)\right)$. Then $t_{1}^{L_{5}} g_{i}\left(a, t_{1}\right) \in T$. For $g_{1}\left(a, t_{1}\right) \leqslant p_{1}\left(t_{1}\right)$ $+h_{1}\left(a, t_{1}\right)$ and $g_{2}\left(-a, t_{1}\right) \leqslant p_{2}\left(t_{1}\right)+h_{2}\left(-a, t_{1}\right)$. But $g_{2}\left(-a, t_{1}\right)=-g_{2}\left(a, t_{1}\right)$, so

$$
-\left(p_{2}\left(t_{1}\right)+h_{2}\left(-a, t_{1}\right)\right) \leqslant g_{2}\left(a, t_{1}\right) \leqslant g_{1}\left(a, t_{1}\right) \leqslant p_{1}\left(t_{1}\right)+h_{1}\left(a, t_{1}\right) .
$$

Thus

$$
\begin{aligned}
-t_{1}^{L_{5}}\left(p_{2}\left(t_{1}\right)+h_{2}\left(-a, t_{1}\right)\right) & \leqslant t_{1}^{L_{5}} g_{2}\left(a, t_{1}\right) \leqslant t_{1}^{L_{5}} g_{1}\left(a, t_{1}\right) \\
& \leqslant t_{1}^{L_{5}}\left(p_{1}\left(t_{1}\right)+h_{1}\left(a, t_{1}\right)\right)
\end{aligned}
$$

and $t_{1}^{L_{5}} g_{i}\left(a, t_{1}\right) \in T$ by Lemma $1(\mathrm{a})$.

Now suppose $g_{1}\left(a, t_{1}\right)$ has a term of the form $\beta t_{1}^{L_{6}} a$. But $t_{1} \geqslant 0$ and all the coefficients of $g_{1}(x, y)$ are in $F^{+}$, so $\left|\beta t_{1}^{L_{6}} a\right| \leqslant\left|g_{1}\left(a, t_{1}\right)\right|$, since this inequality holds in any totally ordered $F-T$ - $T$ bimodule which is a homomorphic image of $R$, and $R$ is a subdirect product of these modules. Thus $\beta\left|t_{1}^{L_{5}} t_{1}^{L_{6}} a\right| \leqslant t_{1}^{L_{5}}\left|g_{1}\left(a, t_{1}\right)\right|=$ $\left|t_{1}^{5} g_{1}\left(a, t_{1}\right)\right|$, and if $N=L_{5}+L_{6}$ then $t_{1}^{N} a \in T$ by Lemma 1(c) (and

$$
\left.N \leqslant M_{1}\left(M_{2}^{n+1}+M_{2}^{n}+\cdots+M_{2}\right)+M_{1}=M_{1}\left(M_{2}^{n+1}+M_{2}^{n}+\cdots+1\right)\right) .
$$

If $N=0$, then $a \in T$ and $t^{N} s a \in T$. If $N \geqslant 1$, then $0 \leqslant t^{N-1} s \leqslant t_{1}^{N}$ and hence $\left|t^{N-1} s a\right|=t^{N-1} s|a| \leqslant t_{1}^{N}|a|=\left|t_{1}^{N} a\right|$; so $t^{N-1} s a \in T$ by Lemma 1(a).

In [7], as part of their characterization of those $f$-rings that can be embedded in unital $f$-rings, Henriksen and Isbell defined an $f$-ring to be infinitesimal if it satisfies the identity $x^{2} \leqslant|x|$ (equivalently $n x^{2} \leqslant|x|$ for each $n \in \mathbf{Z}^{+}$). In [15, Remark, p. 367] we have called an l-ring which satisfies the "dual" identities $n|x| \leqslant\left|x^{2}\right|$ supertesimal. Since the essential use of the nice polynomials $f(x, y)$ in Lemmas 7 and 10 is that " $x \leqslant$ higher powers of $x$ ", we make the following definitions.

A ( $p$-) pseudosupertesimal l-algebra over $F$ is an $l$-algebra $R$ such that for all $a$, $r \in R$, with $r \geqslant 0$ (and $a \geqslant 0$ ), there is a nice polynomial $f(x, y)=-g(x, y)+p(y)$ $+h(x, y)$ in $F[x, y]$ with $f(a, r) \geqslant 0$. A nice polynomial $f(x, y)$ is called $k$ restricted if $h(x, y) \in F\left[x^{k}, y\right] . R$ is a (right) $k$-restricted pseudosupertesimal l-algebra if for all $a, r \in R$ with $r \geqslant 0$ there are two $k$-restricted nice polynomials $f_{1}(x, y)$ and $f_{2}(x, y)$ with $f_{1}(a, r) \geqslant 0, f_{2}(-a, r) \geqslant 0, g_{2}(a, r) \leqslant g_{1}(a, r)$ and $g_{1}(x, y)+$ $g_{2}(x, y)$ has monomials which begin and end in $x\left(g_{1}(x, y)+g_{2}(x, y)\right.$ has a monomial which ends in $x) . R$ is a (right) $p$-k-restricted pseudosupertesimal l-algebra if for all $a, r \in R^{+}$there is a $k$-restricted polynomial $f(x, y)$ with $f(a, r) \geqslant 0$ and $g(x, y)$ has monomials which begin and end with $x$ (which end in $x$ ). Finally, a bounded pseudosupertesimal l-algebra (etc.) is an l-algebra $R$ for which there is an integer $K$ such that for all $a, r \in R$ with $r \geqslant 0$ there is a nice polynomial $f(x, y)$ with $f(a, r) \geqslant 0$ and the degree of $y$ in $g(x, y)$ is $\leqslant K$ and the degree of $h(x, y)$ in $x$ 
is $\leqslant K$. For example, a square archimedean $l$-ring is a bounded $p$-2-restricted pseudosupertesimal $l$-algebra over $\mathbf{Z}$. And a strongly $p$-positive $l$-algebra $R$ is pseudosupertesimal, since if $p(x) \in F^{+}[x]$, then $f(x, y)=p(y-x)$ is a nice polynomial; and if $R$ is unital, then for each element $a$ of $R$ there is a nice polynomial $f(x)=f(x, 1)$ with $f(a) \geqslant 0$; so $R$ is $p$-2-restricted. Also, a commutative $p$-pseudosupertesimal $l$-algebra is $p$-2-restricted. If $R$ is a $P P I l$-algebra with a nice $k$-restricted polynomial $f(x, y)=-g(x, y)+p(y)+h(x, y)$ and $g(x, y)$ has monomials which end in $x$, then $R$ is right $k$-restricted; if $R$ just satisfies $f\left(x^{+}, y^{+}\right)^{-}=0$ then it is right $p$ - $k$-restricted.

We can now give other generalizations of Theorems 1 and 2. The subset $X$ of the $l$-ring $R$ is said to have local (left) $f$-superunits if for each $x \in X$ there is an $e \in T^{+}$ with $|x| \leqslant e|x|$ and $|x| \leqslant|x| e(|x| \leqslant e|x|)$. The element $a \in R$ is regular if $l_{R}(a)=r_{R}(a)=0$.

THEOREM 4. Let $R$ be a pseudosupertesimal torsion-free l-algebra over the totally ordered domain $F$, and suppose that $2 \leqslant k \in \mathbf{Z}$.

(a) If $R$ is right $p$-k-restricted, then $R$ is l-reduced (an l-domain) if and only if it is l-semiprime (l-prime) and $M_{2}=\left\{a \in R^{+}: a^{2}=0\right\}$ has local left $f$-superunits.

(b) If $R$ is right $k$-restricted, then $R$ is reduced if and only if it is l-semiprime and $N_{2}=\left\{a \in R: a^{2}=0\right\}$ has local left $f$-superunits.

Proof. (a) Suppose that $R$ is $l$-semiprime and $a \in M_{2}$ and $e \in T^{+}$with $a \leqslant e a$. Since $a^{k} \in T$ and $a \geqslant 0$ we may use Lemma 10 with $f_{2}(x, y)=-g_{1}(x, y)$. Then $a \leqslant e^{N} a \in T$; hence $a \in T$ by Lemma l(a) and $R$ is $l$-reduced by Lemma 3(a).

The proof of $(b)$ is similar.

THEOREM 5. Let $R$ be a pseudosupertesimal torsion-free l-algebra over the totally ordered domain $F$, and suppose that $k \geqslant 2$. Suppose that $l_{R}(T)=0=r_{R}(T)$ and $R$ is bounded; or $T$ contains a regular element of $R$.

(a) If $R$ is p-k-restricted and l-semiprime (l-prime), then it is l-reduced (an l-domain).

(b) If $R$ is $k$-restricted and l-semiprime, then it is reduced.

Proof. (a) If $a \in M_{2}$ and $t \in T^{+}$, then by Lemma 10 and its right counterpart $t^{N} a$ and $a t^{N}$ are in $T$ for some integer $N$. So if $u \wedge v=0$ in $R$, then $t^{N}(a u \wedge v)=0$ and $(u a \wedge v) t^{N}=0$. If $s \in T$ is regular in $R$, then so is $t=s^{2} \geqslant 0$; so $a \in T$. If $R$ is bounded, then $N$ is independent of $t$ (Lemma 10), so $a u \wedge v \in r_{R}\left(\left\langle T^{N}\right\rangle\right)=r_{R}\left(T^{N}\right)$ by Lemma 5 , and $u a \wedge v \in l_{R}\left(T^{N}\right)$. If we also have $l_{R}(T)=r_{R}(T)=0$, then again $a \in T$. Thus by Lemma 3(a) $R$ is $l$-reduced.

The proof of (b) is similar to that of (a).

From Theorem 4 and Lemma 9(d) we get

COROLlary 3. Let $R$ be a right $k$-restricted $(k \geqslant 2)$ pseudosupertesimal l-algebra over the totally ordered field $F$, and suppose that $R$ is unital with $1 \in R^{+}$. If $R$ is an l-prime i-normal l-algebra, then $R$ is a domain. 
4. The lower $l$-radical. If $\beta(R)$ is the lower $l$-radical of $R$, then since $R / \beta(R)$ is $l$-semiprime, Lemma 3 translates to

LEMMA 11. Let $R$ be an l-ring.

(a) $\beta(R)=\{a \in R:|a|$ is nilpotent $\}=M$ if and only if for $0 \leqslant a \in M$ and $u \wedge v=0$ in $R$, au $\wedge v \in \beta(R)$ and $u a \wedge v \in \beta(R)$. This is true if $M^{+} \subseteq T$.

(b) $\beta(R)=\{a \in R$ : $a$ is nilpotent $\}=N$ if and only if for $a \in N$ and $u \wedge v=0$ in $R,|a| u \wedge v \in \beta(R)$ and $u|a| \wedge v \in \beta(R)$. This is true if $N \subseteq T$.

Lemmas 4, 7 and 10 (and the conditions in Theorems 4 and 5) offer a variety of polynomial characterizations of when $\beta(R)=M$ or $\beta(R)=N$. We record some of these explicitly (as implications). As usual, $R$ is a torsion-free $l$-algebra over the totally ordered domain $F$.

THEOREM 6. Each of the following conditions implies that $\beta(R)=\{a \in R:|a|$ is nilpotent $\}=M \subseteq T$.

(a) $R$ is a right $p$-k-restricted pseudosupertesimal l-algebra for some integer $k \geqslant 2$ and $R$ has local left $f$-superunits.

(b) $R$ is a p-k-restricted pseudosupertesimal l-algebra, with $l_{R}(T)=r_{R}(T)=0$ and $R$ is bounded; or $T$ contains a regular element of $R(k \geqslant 2)$.

(c) Here, we assume $1 \in R^{+}$. If $u \wedge v=0$ with $u$ nilpotent and $v \leqslant 1$, then there is a nice polynomial $f(x, y) \in F[x, y]$ with $f(u, v) \geqslant 0$.

Proof. By Lemma 11(a) we only need that $M^{+} \subseteq T$. For (a) this follows from the argument in Theorem 4(a), and for (b) it follows from the argument in Theorem 5(a). For (c) use Lemma 7(f).

THEOREM 7. Let $R$ be a torsion-free l-algebra over the totally ordered domain $F$. Each of the following conditions implies that $\beta(R)=\{a \in R$ : $a$ is nilpotent $\}=N \subseteq T$.

(a) The square of each element in $R$ is positive; and $R$ has local bi-f-superunits, or $l_{R}(T)=r_{R}(T)=0$.

(b) $R$ is a bounded $k$-restricted pseudosupertesimal l-algebra and $l_{R}(T)=r_{R}(T)=0$ $(k \geqslant 2)$.

(c) $R$ is a $k$-restricted pseudosupertesimal l-algebra and $T$ contains a regular element of $R(k \geqslant 2)$.

(d) $1 \in R^{+}$and $R$ is weakly p-positive.

Proof. By Lemma 11(b) it suffices to show that each nilpotent element is in $T$. For (a) this follows from Lemmas 4 and 6. For (b) and (c) this follows from Lemma 10 (as in the proof of Theorem 5). For (d) it follows from Lemma 7(c).

Since $\beta(R)$ is an $f$-ring (in Theorems 6 and 7) it is the sum of the nilpotent $l$-ideals of $R$ [5, Theorem 3.1]. Let $Z_{n}=\left\{a \in R:|a|^{n}=0\right\}$ and $N_{n}=\left\{a \in R: a^{n}=0\right\}$. If $M_{2}=\left\{a \in R^{+}: a^{2}=0\right\} \subseteq T$, then $Z_{2}(R)=N_{2}(T)$ is an l-ideal of $R$. For if $a \in Z_{2}(R)$, then $|a| \in T$ implies that $a \in T$ since $T$ is a convex $l$-subring. Since $T$ is an $f$-ring (Lemma 1(a)), $\left|a^{2}\right|=|a|^{2}$, and hence $a \in N_{2}(T)$ and $Z_{2}(R)=N_{2}(T)$. By (2), $N_{2}(T)$ is a convex $l$-subgroup of $R$, and then by Lemma $3 Z_{2}(R)=N_{2}(T)$ is an $l$-ideal of $R$. If $M_{2}\left(R / Z_{2}\right) \subseteq T\left(R / Z_{2}\right)$, then $Z_{4}(R)$ is an $l$-ideal of $R$. In particular, 
if $R$ satisfies the hypotheses of (a) or (c) of Theorem 6, then each $Z_{2^{n}}$ is a nilpotent $l$-ideal of index at most $2^{n}$, and $\beta(R)$ is the union of $\left\{Z_{2^{n}}\right\}$.

Similarly, if $N_{2} \subseteq T$, then $N_{2}(R)=N_{2}(T)$ is an l-ideal of $R$; and if $R$ satisfies the hypotheses of (d) or the first part of (a) of Theorem 7, then each $N_{2^{n}}$ is a nilpotent $l$-ideal of index at most $2^{n}$. and $\beta(R)$ is the union of $\left\{N_{2^{n}}\right\}$.

5. Disjoint elements almost commute. Recall that two elements $a$ and $b$ in an $l$-ring $R$ are called disjoint if $a \wedge b=0$.

It is well known that if $a$ and $b$ are two elements in an l-group with $a \wedge b=1$, then $a b=b a$ [3, Theorem 6, p. 295]. Trivially, if $a$ and $b$ are disjoint elements of an $l$-ring which satisfies (3), then $a b=b a$. Examples in $\$ 6$ show that a unital $l$-ring with squares positive need not have this property. However, Theorem 8 gives the appropriate analogue. We first present two lemmas.

An $l$-ring is $l$-simple if it has exactly two $l$-ideals. A unital totally ordered ring is $l$-simple if and only if whenever $a, b>0$ there exist $c, d \geqslant 0$ with $a \leqslant c b d$. Some examples of commutative unital $l$-simple totally ordered rings $F$ are subrings of the reals, totally ordered fields and (commutative) polynomial rings with coefficients in $F$, ordered appropriately. If $R$ is an $l$-algebra over the totally ordered domain $F$, then an algebra $l$-ideal $I$ is closed if $R / I$ is $F$-torsion-free. For an arbitrary algebra $l$-ideal $I, \hat{I}=\{r \in R: \alpha r \in I$ for some $0 \neq \alpha \in F\}$ is the closure of $I$, and $I$ is closed if and only if $I=\hat{I}$.

LEMMA 12. Let $R$ be an l-algebra over the totally ordered domain $F$.

(a) If for each $a \in R^{+}$there exists $e \in R^{+}$with $a \leqslant e a+a e+e a e$, then each l-ideal of $R$ is an algebra l-ideal.

(b) If $F$ is l-simple, then each algebra l-ideal of $R$ is closed.

Proof. (a) If $I$ is an $l$-ideal of $R, a \in I^{+}$and $\alpha \in F^{+}$, then $\alpha a \leqslant \alpha e a+a \alpha e+\alpha e a e$ implies $\alpha a \in I$.

(b) Let $I$ be an algebra $l$-ideal of $R$. If $0<\alpha \in F$ there exists $\beta \in F^{+}$with $1 \leqslant \beta \alpha$. So if $r \in R$ with $\alpha r \in I$, then $|r| \leqslant \beta \alpha|r|=\beta|\alpha r| \in I$; hence $r \in I$.

Diem stated the next lemma for the case that $R$ has squares positive, but, in fact, proved the more general result given here (a proof is also given in [14, p. 199]). It is the motivation for the somewhat surprising lemma which follows it.

LEMMA 13 [5, p. 78]. An l-prime l-ring $R$ is an l-domain if and only if it satisfies the two conditions:

(a) If $a, b \in R^{+}$and $a^{2}=b^{2}=0$, then $a b=0$.

(b) If $a \wedge b=0$ and $a b=0$, then $b a=0$.

The element $a \in R^{+}$is a positive zero-divisor if there is $0 \neq b \in R^{+}$with $a b=0$ or $b a=0$.

LEMMA 14. Let $R$ be a torsion-free l-algebra over the totally ordered domain $F$. Suppose that:

(a) If $a \in R^{+}$and $a^{2}=0$, then $a$ is an f-element of $R$.

(b) If $u \wedge v=0$, with $u$ a positive zero divisor and $v \in T$, then there exists a polynomial $p(x) \in F^{+}[x]$ (of degree $\left.\geqslant 1\right)$ such that $p(v-u) \geqslant 0$; or there is a nice 
polynomial $f(x, y) \in F[x, y]$ with $f(u, v) \geqslant 0$ and $f(x, y)$ has a monomial of degree 1 in $x$ which ends in $x$.

Then if $a, b \in R$ with $a \wedge b=a b=0$, and $e \in T^{+}$, there exists $N \in \mathbf{Z}^{+}$with $e b e^{N} a e=0$.

Proof. We will repeatedly use the fact that $T$ is an $f$-ring (Lemma $1(a))$ and hence it satisfies (4).

Let $e \in T^{+}$and let $a_{1}=a \wedge e$ and $b_{1}=b \wedge e$. We first show that $b e^{m} a_{1} e=0$ for each $m \in \mathbf{Z}^{+}$. Let $b_{2}=b-b_{1}$ and $e_{2}=e-b_{1}$; and let $a_{2}=a-a_{1}$ and $f_{2}=e-a_{1}$. Then by (8) we get

$$
b_{2} \wedge e_{2}=0
$$

and

$$
a_{2} \wedge f_{2}=0
$$

Let $b_{0}=b$ and $a_{0}=a$; then since $a_{1} b_{i}=0$ we have

$$
f_{2} b_{i}=e b_{i} \text { for } 0 \leqslant i \leqslant 2 .
$$

Also, since $a_{i} b_{1}=0$ we get

$$
a_{i} e_{2}=a_{i} e \text { for } 0 \leqslant i \leqslant 2 .
$$

Now $a_{1} \wedge b_{1} e^{m}=0$ and $a_{1}, b_{1} e^{m} \in T$; so $b_{1} e^{m} a_{1}=0$. Also (10) implies $b_{2} e^{m} a_{1}^{l} \wedge e_{2}$ $=0$, for any $l, m \in \mathbf{Z}^{+}$. But $e_{2} \in T$, and $\left(b_{2} e^{m} a_{1}^{l}\right)^{2}=0$ (if $l \geqslant 1$ ) implies $b_{2} e^{m} a_{1}^{l} \in$ $M_{2} \subseteq T$; so

$$
b_{2} e^{m} a_{1}^{l} e=0 \text { for all } m \in \mathbf{Z}^{+} \text {and } l \geqslant 1,
$$

since $b_{2} e^{m} a_{1}^{l} e=b_{2} e^{m} a_{1}^{l} e_{2}=0$, by (13). But then

$$
b e^{m} a_{1} e=\left(b_{2}+b_{1}\right) e^{m} a_{1} e=b_{2} e^{m} a_{1} e+b_{1} e^{m} a_{1} e=0 .
$$

By (11) $b_{1} e^{m} a_{2} \wedge f_{2}=0$, and therefore by (12)eb $e e^{m} a_{2}=f_{2} b_{1} e^{m} a_{2}=0$. So

$$
e b e^{m} a e=e b_{2} e^{m} a_{2} e \text { for all } m \in \mathbf{Z}^{+},
$$

since $e b_{1} e^{m} a_{2}=b e^{m} a_{1} e=0$ and

$$
e b_{2} e^{m} a_{2} e=e\left(b-b_{1}\right) e^{m}\left(a-a_{1}\right) e=e b e^{m} a e-e b e^{m} a_{1} e-e b_{1} e^{m} a_{2} e .
$$

Since $\left(b_{2}\left(f_{2} e\right)^{m} a_{2}\right)\left(f_{2} e\right)^{s} \in M_{2} T^{+} \subseteq T^{+}$we get

$$
b_{2}\left(f_{2} e\right)^{m} a_{2}\left(f_{2} e\right)^{s} a_{2} \wedge f_{2}=0
$$

by (11); and hence (12) implies

$$
e b_{2}\left(f_{2} e\right)^{m} a_{2}\left(f_{2} e\right)^{s} a_{2}=0 \text { for all } m, s \in \mathbf{Z}^{+} .
$$

Let $p(x)$ be a polynomial in $F[x]$ of degree $\geqslant 1$ and with positive coefficients such that $p\left(f_{2} e-a_{2}\right) \geqslant 0$. Then

$$
0 \leqslant \alpha_{0}+\alpha_{1}\left(f_{2} e-a_{2}\right)+\cdots+\alpha_{n}\left(f_{2} e-a_{2}\right)^{n}=p\left(f_{2} e-a_{2}\right)
$$

and so $\left(\alpha_{0}=0\right.$ if $\left.1 \notin R^{+}\right)$

$$
0 \leqslant g\left(a_{2}, f_{2} e\right) \leqslant \alpha_{0}+\sum_{k \geqslant 1} \alpha_{k}\left(f_{2} e\right)^{k}+h\left(a_{2}, f_{2} e\right)
$$


where $-g\left(a_{2}, f_{2} e\right)$ is the sum of all those monomials in $a_{2}$ and $f_{2} e$ in (17) which contain just one $a_{2}$, and $h\left(a_{2}, f_{2} e\right)$ is the sum of all those monomials which contain more than one $a_{2}$. A typical term in $h\left(a_{2}, f_{2} e\right)$ is of the form $\alpha w=$ $\alpha\left(f_{2} e\right)^{m_{1}} a_{2}\left(f_{2} e\right)^{m_{2}} a_{2} \cdots\left(f_{2} e\right)^{m_{t}}$ with $m_{i} \in \mathbf{Z}^{+}, t \geqslant 3$ and $\alpha \in F$. By (16) $e b_{2} w=0$ and hence $e b_{2} h\left(a_{2}, f_{2} e\right)=0$. From (18) we get

From (18) we get

$$
0 \leqslant e b_{2} g\left(a_{2}, f_{2} e\right) \leqslant \sum \alpha_{k} e b_{2}\left(f_{2} e\right)^{k} .
$$

A typical term in $g\left(a_{2}, f_{2} e\right)$ is $\alpha\left(f_{2} e\right)^{m} a_{2}\left(f_{2} e\right)^{s}$. But

$$
b_{2}\left(f_{2} e\right)^{m} a_{2}\left(f_{2} e\right)^{s} e \wedge b_{2}=0 \text { for all } m, s \in \mathbf{Z}^{+},
$$

since $f_{2} \leqslant e$ and

$$
\begin{aligned}
0 & \leqslant b_{2}\left(f_{2} e\right)^{m} a_{2}\left(f_{2} e\right)^{s} e \wedge b_{2} \leqslant b_{2}\left(f_{2} e\right)^{m} a_{2}\left(e^{2}\right)^{s} e \wedge b_{2} \\
& =b_{2}\left(f_{2} e\right)^{m} a_{2} e_{2} e^{2 s} \wedge b_{2}=0
\end{aligned}
$$

by (13) and (10); and (20) implies

$$
e b_{2}\left(f_{2} e\right)^{m} a_{2}\left(f_{2} e\right)^{s} e \wedge e b_{2}\left(f_{2} e\right)^{k} e=0 \quad \text { for all } m, s, k \in \mathbf{Z}^{+} .
$$

Now (19), (21) and (7) imply that

$$
0 \leqslant e b_{2} g\left(a_{2}, f_{2} e\right) e=e b_{2} g\left(a_{2}, f_{2} e\right) e \wedge \sum \alpha_{k} e b_{2}\left(f_{2} e\right)^{k} e=0,
$$

and hence

$$
e b_{2} g\left(a_{2}, f_{2} e\right) e=0 .
$$

However, one term in $g\left(a_{2}, f_{2} e\right)$ is $\alpha\left(f_{2} e\right)^{m} a_{2}$ with $0<\alpha \in F$ and $m \geqslant 0$; since $g(x, y) \in F^{+}[x, y],(22)$ implies

$$
e b_{2}\left(f_{2} e\right)^{m} a_{2} e=0 .
$$

Now for any $k \in \mathbf{Z}^{+}$

$$
b_{2}\left(f_{2} e\right)^{k} a_{2}=b_{2}\left(e-a_{1}\right) e\left(e-a_{1}\right) e \cdots\left(e-a_{1}\right) e a_{2}=b_{2} e^{2 k} a_{2},
$$

since all other terms contain a factor $b_{2} e^{r} a_{1}^{l} e$ with $l \geqslant 1$, and $b_{2} e^{r} a_{1}^{l} e=0$ by (14). Thus

$$
e b e^{2 m} a e=e b_{2} e^{2 m} a_{2} e=e b_{2}\left(f_{2} e\right)^{m} a_{2} e=0
$$

by (15), (24) and (23).

If there is a nice polynomial $f(x, y)=-g(x, y)+p(y)+h(x, y)$ with $f\left(a_{2}, f_{2} e\right)$ $\geqslant 0$, then we again get (18) (some $\alpha_{k}$ may be negative); and if $g(x, y)$ has a monomial which ends in $x$, the calculation from (18) through (25) is still valid.

COROLlary 4. Suppose that $R$ satisfies the hypotheses of Lemma 14, and it has local left (right) $f$-superunits and $l_{T}(T)=0\left(r_{T}(T)=0\right)$. Then $a \wedge b=a b=0$ implies $b a=0$.

Proof. If $e \in T^{+}$is a left superunit for $\{a, b\}$, then by Lemma $140 \leqslant b a e \leqslant$ $e b e^{N} a e=0$ for some $N$. If $t \in T^{+}$, then $e+t$ is also a left superunit for $\{a, b\}$; so $b a(e+t)=0$ and hence $b a t=0$. Since $l_{T}(T)=0, b a=0$. 
The $F$-l-algebra $R$ is called (right) weakly p-pseudosupertesimal if whenever $u \wedge v$ $=0$ in $R$ there exists a nice polynomial $f(x, y)=-g(x, y)+p(y)+h(x, y) \in$ $F[x, y]$ (such that $g(x, y)$ has a monomial ending in $x$ ) and $f(u, v) \geqslant 0$. Note that this is a one variable constraint since $u=a^{+}$and $v=a^{-}$for $a=u-v$.

THEOREM 8. Let $R$ be a torsion-free l-algebra over the totally ordered domain $F$, and suppose that $R$ has local $f$-superunits. Each of the following statements implies that the closed l-ideals of $R$ generated by $a b$ and ba are identical whenever $a \wedge b=0$ in $R$.

(a) $R$ has square positive.

(b) $R$ is unital and strongly p-positive.

(c) $R$ is unital and right weakly p-pseudosupertesimal.

(d) $R$ is right p-k-restricted pseudosupertesimal with $k \geqslant 2$.

Proof. We first note that the hypotheses are satisfied by each homomorphic image $R^{*}$ of $R$ (for (c) use (9)). Let $I$ be the $l$-ideal of $R$ generated by $a b ; I$ is an algebra $l$-ideal by Lemma 12(a), with closure $\hat{I}$. If $R^{*}=R / \hat{I}$, then, in each case, we have seen that $M_{2}^{*}=M_{2}\left(R^{*}\right) \subseteq T^{*}=T\left(R^{*}\right)$. For (a) use Lemma 4; for (b) use Lemma 7(d) (or the fact that (b) implies (c)); for (c) use Lemma 7(f); for (d) use Lemma 10. Since $a^{*} \wedge b^{*}=a^{*} b^{*}=0, b^{*} a^{*}=0$ by Corollary 4. So $b a \in \hat{I}$, and similarly, $a b$ is in the closed $l$-ideal of $R$ generated by $b a$.

It is possible to strengthen Theorem $8(\mathrm{~b})$ by assuming weakly $p$-positive and the following. Let $p(x)=p_{1}(x)-p_{2}(x)$ where $p_{1}(x)$ (respectively, $\left.-p_{2}(x)\right)$ is the sum of the terms of $p(x)$ with a positive (respectively, negative) coefficient. Then for each $a \in R$ we require $p(x)=p_{1}(x)-p_{2}(x) \in F[x]$ with $p(a) \geqslant 0, p(1)-p(0)>0$ in $R$, and for each $i \geqslant 0, \gamma_{i}=\Sigma_{k \geqslant i+1}\left(\alpha_{k}-\beta_{k}\right) \geqslant 0\left(\alpha_{k}\right.$ and $\beta_{k}$ are the coefficients of $x^{k}$ in $p_{1}(x)$ and $\left.p_{2}(x)\right)$. Now the proof of Lemma 14 goes through with $e=1$. For $b_{2} f_{2}=b_{2}\left(1-a_{1}\right)=b_{2}$ by (14), and hence in (19) $b_{2} g\left(a_{2}, f_{2}\right)=\sum_{i \geqslant 0} \gamma_{i} b_{2} a_{2} f_{2}^{i}$; so the argument after (19) is still valid.

6. Examples and a remark. Let $R$ be a torsion-free $l$-algebra over the totally ordered domain $F$. In [14, Theorem 8] it is shown that the following statements are equivalent if $R$ has a left $f$-superunit $e$ :

(i) $R$ satisfies $x^{+} x^{-}=0$.

(ii) If $a \wedge e=0$, then $a=0$.

(iii) If $a \geqslant 0$ and $a \wedge e$ is nilpotent, then $a \in T$.

(iv) If $a \geqslant 0$ and $(a \wedge e)^{2}=0$, then $a \in T$.

(v) $R$ has squares positive and

(26) If $a \in R^{+}$and $(a \wedge e)^{2}=0$, then $a^{2}=0$.

(vi) Assume $e=1 . R$ is a $P P I$ l-algebra with a polynomial $p(x)$ which satisfies (26).

In fact, it is easily seen that (iv) is equivalent to

(vii) $M_{2}=\left\{a \in R^{+}: a^{2}=0\right\} \subseteq T$ and $R$ satisfies (26).

Thus, to get other equivalences, each of the polynomial constraints which generalize squares positive or $x^{+} x^{-}=0$ and implies $M_{2} \subseteq T$ can be substituted for "squares positive" in (v). Hence, these constraints are not that far removed from their squares positive origin. 
EXAMPLE 1. A commutative, unital, reduced, $i$-normal, weakly $p$-positive $l$-domain in which all the idempotents are positive, but which is not a domain (see [4, Example 9f (II), p. 48]).

Let $\bar{R}=\mathbf{Q} \oplus \mathbf{Q}$ be the (ring) direct sum of two copies of the rationals with positive cone $\bar{R}^{+}=\{(u, v): 0 \leqslant v \leqslant u\}$ and let

$$
R=\{(2 n, 2 m)+(k, k): n, m, k \in \mathbf{Z}\} \text {. }
$$

Then $\bar{R}$ is an $l$-domain and if $a=(u, v) \in \bar{R}$, then either $p(a) \geqslant 0$ or $p(a) \leqslant 0$, where $p(x)=v x-x^{2}$; so $R$ is an $i$-normal $p$-positive $l$-algebra over $\mathbf{Z}$.

The following table shows that $R$ is weakly $p$-positive.

TABLE 1

$$
\begin{aligned}
& \quad \underline{a=(u, v) \in \mathbf{Z} \times \mathbf{Z}} \\
& a \in \bar{R}^{+} \cup-\bar{R}^{+} \cup\{(u, 1): u<0\} \\
& u<0 \text { and } v \geqslant 2 \\
& u<0 \text { and } v<u \\
& u=0 \text { and } v<2 \\
& u=0 \text { and } v=2 \\
& u=0 \text { and } v>2 \\
& u>0 \text { and } v<0 \\
& u>0 \text { and } v>u
\end{aligned}
$$

$$
\begin{gathered}
p(x) \text { with } p(a) \in \bar{R}^{+} \text {and } p^{\prime}(1)>0 \\
\hline p(x)=x^{2} \\
p(x)=v x^{2}-x^{3} \\
p(x)=-v x^{2}+x^{3} \\
p(x)=x^{2}-v x \\
p(x)=2 x+x^{2}-x^{3} \\
p(x)=v x-x^{2} \\
p(x)=x^{2}-v x \\
p(x)=v^{3} x-x^{4}
\end{gathered}
$$

EXAMPLE 2. A unital $l$-ring with squares positive in which disjoint elements do not commute.

An example is given by the free algebra generated by the set $X$. Let $\Delta$ be the free semigroup (with identity $e$ ) generated by $X$, and let $Y$ be the set $X$ together with a total order. If $s=x_{1} x_{2} \cdots x_{p} \in \Delta$, then $s$ is said to have length $p: l(s)=p$. We make $\Delta$ into a partially ordered semigroup by defining, for $s, t \in \Delta, s<t$ if

(i) $1 \leqslant l(s)<l(t)$ or

(ii) $s=x_{1} \cdots x_{m} x_{m+1} \cdots x_{p}, t=x_{1} \cdots x_{m} y_{m+1} \cdots y_{p}, p \geqslant 2$, and $x_{m+1}<y_{m+1}$ in $Y$ for some $m \geqslant 0$.

In this ordering the set $X \cup\{e\}$ is trivially ordered and is at the "bottom" of $\Delta$, whereas the elements of length $\geqslant 2$ form a chain above $X$. Let $R=A[\Delta]=\{f=$ $\left.\Sigma a_{s} s: s \in \Delta, a_{s} \in A\right\}$ be the semigroup ring of $\Delta$ over the totally ordered domain $A$. By the support of an element $f=\Sigma a_{s} s$ in $R$ we mean $\left\{s \in \Delta: a_{s} \neq 0\right\}$. If $R$ is given the positive cone $R^{+}=\left\{f=\Sigma a_{s} s: a_{s}>0\right.$ if $s$ is a maximal element in the support of $f$ \}, then $R$ is a unital $l$-ring with squares positive (this may be verified directly or it follows from [16, Theorem I(b) and Lemma 2]). If $X$ has at least two elements and if $x$ and $y$ are distinct in $X$, then $x \wedge y=0$ in $R$, but $x y \neq y x$. Another such example is obtained by strengthening the order of $\Delta$ slightly by adding

(iii) $e<t$ if $l(t) \geqslant 2$.

We also note that, if in (i) and (iii) we stipulate that $l(t) \geqslant 2 n$, and if we require that $p \geqslant 2 n$ in (ii), for a fixed positive integer $n$, then $R$ will satisfy $\left(x^{2 n}\right)^{-}=0$ but not $\left(x^{m}\right)^{-}=0$ for $m<2 n$. 
The referee has supplied the following simpler example (any example must take into account [15, Theorem 1] and the equivalence of (i) and (ii) in the first paragraph of this section).

EXAMPLE 3. Let $\theta$ be a nontrivial order preserving automorphism of the totally ordered field $F$. Let $F[x ; \theta]$ be the twisted polynomial ring determined by $\theta$. So the elements of $F[x ; \theta]$ are polynomials $p(x)=a_{0}+a_{1} x+\cdots+a_{n} x^{n}$ where $a_{i} \in F$. The elements of $F[x ; \theta]$ are added as usual and multiplied like polynomials subject to the commutation rule $x a=(a \theta) x$ for any $a \in F$. Let $p(x)>0$ if $n \geqslant 2$ and $a_{n}>0$, and let $a_{0}+a_{1} x \geqslant 0$ if $a_{0} \geqslant 0$ and $a_{1} \geqslant 0$. Then squares in $F[x ; \theta]$ are positive; $a \wedge x=0$ for any $a \in F$, and $a x \neq x a$ if $a \theta \neq a$.

\section{REFERENCES}

1. A. Bigard, Contribution à la théorie des groupes réticulés, Thèse Sci. Math., Paris, 1969.

2. A. Bigard, K. Keimel and S. Wolfenstein, Groupes et anneaux réticulés, Lecture Notes in Math., vol. 608, Springer-Verlag, Berlin and New York, 1977.

3. G. Birkhoff, Lattice theory, 3rd ed., Amer. Math. Soc. Colloq. Publ., vol. 25, Amer. Math. Soc, Providence, R.I., 1968.

4. G. Birkhoff and R. S. Pierce, Lattice-ordered rings, An. Acad. Brasil. Ci. 28 (1956), 41-69.

5. J. E. Diem. A radical for lattice-ordered rings, Pacific J. Math. 25 (1968), 71-82.

6. L. Fuchs, Partially ordered algebraic systems, Pergamon Press, New York, 1963.

7. M. Henriksen and J. Isbell, Lattice-ordered rings and function rings, Pacific J. Math. 12 (1962), 533-565.

8. I. N. Herstein, Noncommutative rings, Carus Math. Monographs 15 (1968).

9. D. G. Johnson, A structure theory for a class of lattice-ordered rings, Acta Math. 104 (1960), 163-215.

10. R. S. Pierce, Radicals in function rings, Duke Math J. 23 (1956), 253-261.

11. M. A. Shatalova, $l_{A}$ and $l_{I}$ rings, Siberian Math. J. 7 (1966), 1084-1094.

12. H. J. Shyr and T. M. Viswanathan, On the radicals of lattice-ordered rings, Pacific J. Math. 54 (1974), 257-260.

13. S. A. Steinberg, Finitely-valued f-modules, Pacific J. Math. 40 (1972), 723-737.

14. __ Identities and nilpotent elements in lattice ordered rings, Ring Theory (S. K. Jain, ed.), Dekker, New York, 1977.

15. On lattice-ordered rings in which the square of every element is positive, J. Austral. Math. Soc. 22 (1976), 362-370.

16. __ Examples of lattice-ordered rings, J. Algebra 72 (1981), 223-236.

DePartment of Mathematics, University of TOledo, TOledo, OHIO 43606 